\title{
Ein neues Konzept zur Bewertung von nachhaltigem Zugang zu Wasser
}

\author{
Auch nach der Anerkennung eines Rechts auf \\ Wasser durch die UN-Generalversammlung im \\ Jahr 2010 steht ein Durchbruch bei dessen \\ Umsetzung immer noch aus. Dazu fehlen bis- \\ her nicht zuletzt ein klares Begriffskonzept und \\ Prüfsteine, wann das Recht auf Wasser erfüllt \\ sein soll. Der Beitrag entwirft auf institutionen- \\ ökonomischer Grundlage ein neues, nachhaltig- \\ keitsorientiertes Bewertungssystem für den \\ Zugang zu Wasser. \\ Von Erik Gawel und Wolfgang Bretschneider
}

O bwohl auf der Ebene internationaler Deklarationen das Recht auf Wasser (RaW) allgemein etabliert ist, bleibt seine konkrete Umsetzung doch im weltweiten Maßstab weiterhin stark defizitär (Meier et al. 2013; Meier et al. 2014; Flores et al. 2013; Singh 2013).

Die flächendeckende Implementation eines RaW, das begrifflich auf einen angemessenen "Zugang“ zu Wasser abstellt, kann aber nur gelingen, wenn zunächst einmal eine praxistaugliche Konkretisierung des darauf gestützten Anspruchs vorliegt (Dubreuil 2006; Sacher/Windfuhr 2008): Unter welchen Umständen ist also ein „Zugang“ zu Trinkwasser gegeben bzw. ein darauf gerichtetes RaW verletzt? Bislang werden dazu zwei Bewertungskriterien diskutiert: die Zumutbarkeit (Sacher/Windfuhr 2008; CESCR 2002; Roaf et al. 2005; Gorsboth/Wolf 2008; Albuquerque 2011; Murthy 2013) und die Diskriminierungsfreiheit (CESCR 2002; Roaf et al. 2005; Albuquerque 2011; WHO/UNICEF 2012; Albuquerque/Roaf 2012) des Zugangs zu Trinkwasser.

\section{Konzeptionelle Defizite als Implementationsbremse}

Dies aber ist unvollständig, da es bislang nicht gelungen ist, auch das Anliegen der Nachhaltigkeit, das heißt der langfristigen ökologischen und ökonomischen Tragfähigkeit der Wasserversorgung, in den Zugangsbegriff zu integrieren. Eine zielführende Bewertung von Versorgungssituationen kann nämlich nur dann gelingen, wenn dabei auch die Nachhaltigkeitsleistungen der dem Zugang potenziell entgegenstehenden Hürden (pekuniäre, räumliche, zeitliche und qualitative Hür- den) mit einbezogen werden. Diese Zugangshürden, wie etwa der zu zahlende Wasserpreis oder die Raumüberwindung beim Wasserholen, sind nicht etwa im Interesse eines RaW vollständig zu beseitigen, obgleich sie dem Nutzer in der Tat Lasten aufbürden und ihn tendenziell von der Wassernutzung abhalten. Vielmehr haben diese Hürden auch eine wichtige Funktion, und zwar genau insoweit, als sie ökologischen und ökonomischen Nachhaltigkeitszielen der Wasserversorgung (Umweltschutz, Effizienz, Finanzierung) angemessen Raum geben (OECD 2010).

Denn nur wo Wasserressourcen nachhaltig geschützt werden, eine Wasserinfrastruktur auskömmlich finanziert ist und zugleich verschwendungsfrei arbeitet, lässt sich auch langfristig ein RaW sicherstellen. Der Schlüssel zu einer nachhaltigen Umsetzung eines RaW liegt daher in der Analyse des komplexen Gefüges unterschiedlicher Zugangshürden.

\section{Millennium Development Goals und Sustainable Development Goals}

In den vergangenen Jahren wurde auf globaler Ebene die Umsetzung eines RaW besonders im Rahmen der Millennium Development Goals (MDGs) aus dem Jahr 2000 vorangetrieben. In ihnen wurde das Ziel formuliert, ,[to] [h]alve, by 2015, the proportion of the population without sustainable access to safe drinking water and basic sanitation“ (UN 2015). Dieses Ziel wurde nach eigenem Bekunden bereits vorzeitig 2010 erreicht (UNICEF/WHO 2015). Allerdings bestehen mindestens zwei Probleme: Erstens verbleibt immer noch ein Anteil der Weltbevölkerung, dem es an einem derart definierten Zugang mangelt. Und zweitens hat die dabei verwendete Konzeption von „Zugang“ Kritik auf sich gezogen: So wurde „Zugang“ im Rahmen der MDGs an bestimmten Darreichungsformen (,improved water sources“, zum Beispiel Hausnetzanschluss, Regenwassersammlung) festgemacht, kann damit aber die graduelle Problematik eines nur teilweise oder in einzelnen Facetten erfüllten Zugangs nicht einfangen (Bartram 2008).

Im September 2015 wurden nun mit dem Folgeprojekt der Sustainable Development Goals (SDGs) die Weichen für die nächsten 15 Jahre gestellt, in denen wiederum der Zugang zu Wasser ein zentrales Anliegen ist. In den SDGs wird das einschlägige Ziel 6 nun so formuliert: „Ensure availability and sustainable management of water and sanitation for all." Gemäß dem Unterziel 6.1 soll bis 2030 nunmehr für alle Menschen der Zugang zu Trinkwasser (,universal and equitable access“) gewährleistet sein (UN 2015). 


\section{Konzeptionelle Fortschritte weiterentwickeln}

Mit Blick auf die Messung der konkreten Umsetzung kann man durchaus auf konzeptionelle Fortschritte der letzten 15 Jahre beim Zugangskonzept zurückgreifen. So wurde klargestellt, dass es sich bei „Zugang“ stets um ein graduelles Problem handelt (Howard/Bartram 2003). Das bedeutet, dass Zugang kein dichotomes Merkmal ist, das unter Verweis auf einen starren Katalog an Darreichungsformen von Wasser entweder voll gewährleistet oder nicht gewährleistet wäre. Ferner wurde spätestens mit dem General Comment No. 15 (GC15) des „Committee on Economic, Social and Cultural Rights“ implizit deutlich, dass es sich zugleich um ein mehrdimensionales Problem handelt (Roaf et al. 2005): Zugang zu Trinkwasser hat eine räumliche, eine zeitliche, qualitative und nicht zuletzt pekuniäre Dimension. Wesentlicher Bestandteil des GC15 ist es, für jede Dimension ein bestimmtes Kriterium auszumachen: Benannt werden die physical accessibility (räumlich), availability (zeitlich) und acceptability (qualitativ) sowie die affordability (pekuniär). Diese Kriterien lassen sich als individuelle Zumutbarkeitskriterien des Zugangs zusammenfassen und haben die Diskussion zur Umsetzung sehr belebt und differenziert (Thielbürger 2015). Damit haben sie zugleich die Debatte stark von einem naiv-absoluten hin zu einem aufgeklärten-relativen Verständnis eines RaW geführt. Neben diesen $\mathrm{Zu}$ mutbarkeitskriterien wurde bereits im GC15 und in den letzten Jahren noch einmal verstärkt mit der interindividuellen Nichtdiskriminierung (keine sachgrundlose Ungleichbehandlung) ein zusätzliches Kriterium verstärkt diskutiert.

Diese Kriterien der Zumutbarkeit und Diskriminierungsfreiheit wurden auch im Rahmen der SDG-Vorbereitung berücksichtigt. Allerdings gelingt es so weiterhin nicht, auch das Anliegen der Nachhaltigkeit in den Zugangsbegriff zu integrieren. Auf diesen Aspekt aber legen die SDGs gerade im Gegensatz zu den MDGs, die sich allein auf soziale Ziele konzentrierten, besonderen Wert ( $\mathrm{Lu}$ et al. 2015, u. a. integriertes Wasserressourcen-Management [Unterziel 6.5], water use efficiency [6.4]). Und auch die RaW-Literatur der letzten Jahre hat in Anbahnung der SDGs verstärkt formuliert, dass das RaW ökologisch, aber auch ökonomisch nachhaltig umgesetzt werden soll (Albuquerque/Roaf 2012; Baillat et al. 2013; UNESCO 2015).

\section{Ein neues Konzept eines „nachhaltigen Zugangs“}

Vor diesem Hintergrund ist unsere Ausgangsthese, dass die konzeptionelle Fundierung für einen nachhaltigen Zugangsbegriff bisher noch nicht gelungen ist. Wir versuchen, eine solche Fundierung mithilfe institutionenökonomischer Konzepte zu liefern und bauen dabei auf den erwähnten bisherigen konzeptionellen Weiterentwicklungen der RaW-Debatte auf.

Zunächst sei betrachtet, was man unter den Nachhaltigkeitszielen der Wasserversorgung verstehen soll. Es handelt sich dabei um die Wirtschaftlichkeit der Bereitstellung von Wasser-

\section{„Mit der anstehenden Verabschiedung der Sustainable development goals muss auch die konzeptionelle Entwicklung eines ,Rechts auf Wasser" fortgeschrieben werden."}

diensten (ökonomische Effizienz), die ökologische Nachhaltigkeit des Zugriffs auf Wasserressourcen und die Refinanzierung von Wasserdiensten (OECD 2010). Diese Nachhaltigkeitsziele stehen kurzfristig durchaus in einem Konkurrenzverhältnis zum RaW. Sie lassen sich aber nicht dem RaW absolut unterordnen. Denn erstens ist nicht jede Wassernutzung in privaten Haushalten grundrechtlich schützenswert (zum Beispiel swimming pools). Zweitens repräsentieren die konkurrierenden Ziele andere, zum Teil grundrechtlich schützenswerte Wassernutzungen (zum Beispiel Nahrungsmittelproduktion, Schutz der Umwelt). Drittens kann das RaW selbst nur dann nachhaltig umgesetzt werden, wenn diese konkurrierenden Belange in gewissem Umfange zur Geltung kommen. Denn nur wo Wasserressourcen nachhaltig geschützt werden, eine Wasserinfrastruktur auskömmlich finanziert ist und verschwendungsfrei arbeitet, lässt sich auch langfristig ein RaW sicherstellen. Auf diese Weise wird deutlich, dass sich in der langfristigen Perspektive die Zielkonkurrenz zwischen dem RaW und der Nachhaltigkeit stark abschwächt oder sogar umkehrt.

Es erscheint daher ein Zugangsbegriff nötig, der das Spannungsverhältnis zwischen dem RaW als sozialem Ziel und den kurzfristig konkurrierenden, übrigen nachhaltigkeitsbezogenen Zielen der Wasserversorgung verarbeiten kann und insoweit auch das RaW selbst auf Nachhaltigkeit hin programmiert. Die Institutionenökonomik kann eine solche Perspektive anbieten, indem der Zugang gleichsam von seiner Negation her definiert wird: Was steht dem Zugang jeweils entgegen? Damit werden die Hürden des Zugangs zum relevanten Analysegegenstand. Im Gegensatz zum abstrakten „Zugang“ sind die Hürden besser beobachtbar und greifbar und werfen überhaupt erst das Umsetzungs-Problem eines RaW konkret auf. Diese Zugangshürden lassen sich aufgliedern in die Geldausgaben als pekuniäre Hürde sowie in drei nicht pekuniäre Hürden (räumlich, zeitlich, qualitativ): Ein Nutzer muss nicht nur u. U. eine räumliche Distanz überwinden, um an Wasser zu gelangen (UNESCO 2015), sondern gegebenenfalls. auch auf Wasser warten (zeitliche Hürde) oder hygienisch nicht einwandfreies Wasser zum Teil selbst aufbereiten (qualitative Hürde). Ein Wasserversorger kann nun durch Leistungen wie Wasseraufbereitung, -speicherung und -transport diese Hürden senken (Balance/Taylor 2005). Damit steigt zwar der ser- 
vice level, allerdings umgekehrt auch die pekuniäre Hürde, also der Preis für Wasserdienstleistungen. Zwischen den unterschiedlichen Hürdenarten bestehen also umfangreiche Wechselwirkungen.

Die Intuition des RaW ist es, zur Umsetzung seines Anspruchs alle Hindernisse (Hürden) gänzlich aus dem Weg zu räumen. Das aber würde bedeuten, dass jeder Haushalt jederzeit unbegrenzt und kostenlos über qualitativ höchstwertiges Wasser frei Haus verfügen könnte. Das aber verbietet sich aus Gründen der Nachhaltigkeit, da Hürden mit Blick auf eine nachhaltige Wasserversorgung wichtige Funktionen erfüllen. Insbesondere begrenzen Hürden in gewissem Maße den Wasserkonsum (Exklusionsfunktion).

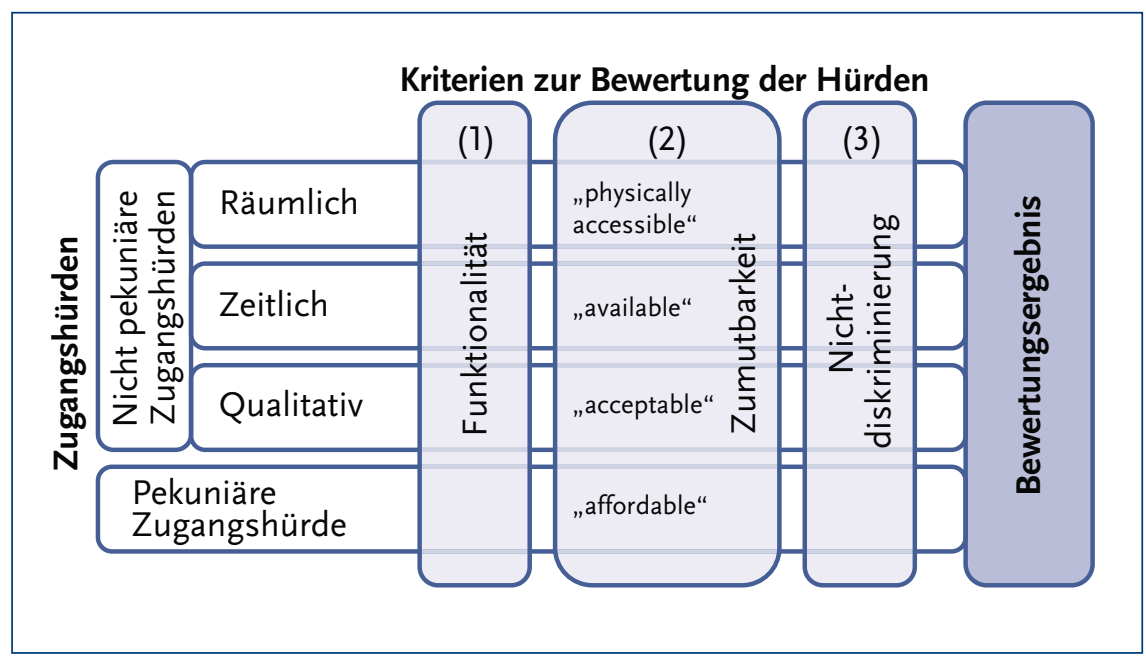

Abbildung 1: Zwölf-Punkt-Prüfmatrix aus den Hürden der vier access-Dimensionen und den drei normativen Kriterien
Angesichts der ökonomischen Knapp-

heit der natürlichen Ressource Wasser und der zugehörigen Dienste muss eine Wasserwirtschaft ohnehin in „irgendeiner Form“ rationieren, da nicht alle Ansprüche bezüglich Menge, Qualität, Ort und zeitlicher Verfügbarkeit gleichermaßen maximal bedient werden können. In ihrer Exklusionsfunktion schützen Hürden also vor Verschwendung; dies kommt gerade anderen Wassernutzern (zum Beispiel für Lebensmittelproduktion) und nicht zuletzt den aquatischen Ökosystemen zugute. Pekuniäre Zugangshürden sorgen zudem für eine auskömmliche, das heißt auch nachhaltige Finanzierung jener Dienstleistungen, welche die nicht pekuniären Hürden durch ihr Service-Angebot senken (Finanzierungsfunktion) (Gawel/Bretschneider 2012). Analog trägt der Nutzer durch Überwindung der nicht pekuniären Hürden (durch Warten, Holen, Speichern, Abkochen etc.) selbst einen Teil der Last dafür, dass das Wasser zur eigenen Nutzung an einem bestimmten Ort zu einer bestimmten Zeit in einer bestimmten Qualität verfügbar gemacht wird. Er übernimmt insoweit teilweise mögliche Leistungen eines Versorgers durch hauswirtschaftliche Produktion (Substitutionsfunktion) (Becker 1965), was in einem bestimmten Umfang (zum Beispiel aus Kostengründen) sinnvoll sein kann.

\section{Zugangshürden als Schlüssel zum Verständnis}

Aus dieser Perspektive muss man nun die Frage nach der Umsetzung eines RaW anders stellen: Unter welchen Umständen kann man die Hürden beim Wasserzugang in Art und Umfang als angemessen und deshalb auch als legitim betrachten? Dies ist der Fall, soweit drei Kriterien erfüllt sind: Die Hürden sollen erstens funktional sein. Das bedeutet, dass sie illegitim sind, soweit sie gerade keine Nachhaltigkeitsfunktion erfüllen. Das betrifft bei pekuniären Hürden insbesondere die Preisaufschläge von Monopolisten, aber auch die preistreibende Wirkung von Ineffizienzen öffentlicher Versorger. Bei räumlichen
Hürden etwa betrifft dies Gefahren für Leib und Leben beim Wasserholen. Diese „Funktionalität“ von Zugangshürden lässt sich grundsätzlich wissenschaftlich bestimmen.

Die weiteren Kriterien sind die Zumutbarkeit und NichtDiskriminierung, die bereits Gegenstand der RaW-Debatte sind. Hier wird aber deutlich, dass auch sie sich ihrem Wesen nach auf die Hürden beziehen dürften. Die genannten „-ilities“ des GC15 (availability usw.) beschreiben nämlich die Fähigkeit des Haushalts, die Hürden soweit überwinden zu können, dass er „hinreichend“ Wasser konsumieren kann und sich dabei nicht zu stark an anderen Stellen einschränken muss. Im Übrigen wird mit den Zumutbarkeitskriterien des GC15 bereits implizit die Legitimität von Zugangshürden anerkannt. Verbreitete statistische Zumutbarkeitsmaße wie der Quotient aus Wasserausgaben und Einkommen zur Bestimmung der affordability sind allerdings wissenschaftlich höchst umstritten (Hancock 1993; Gawel et al. 2013). In jedem Fall muss die Zumutbarkeit letztlich politisch bestimmt werden. Klare „objektive“ Zumutbarkeitsgrenzen sind wissenschaftlich nicht leistbar. Das Kriterium der Diskriminierungsfreiheit bedeutet schließlich, dass keine unbegründeten (willkürlichen) Unterschiede hinsichtlich Höhe und Art der Hürden zwischen unterschiedlichen Nutzern bestehen sollen (WHO/UNICEF 2012; Albuquerque/Roaf 2012). Auch diese normative Setzung muss grundsätzlich politisch bestimmt werden.

\section{Acht Fälle für ein Recht auf Wasser}

Die Zugangshürden zu Wasser müssen sich deshalb an drei (anstelle der bisherigen zwei) Prüfkriterien messen lassen: individuelle Zumutbarkeit, Diskriminierungsfreiheit und Funktionalität. Um nun die Einhaltung des RaW zu prüfen, ist prinzipiell jedes der drei Kriterien auf jede der vier Hürdenarten anzuwenden. Es sind mithin zwölf (Teil-)Prüfungen vorzunehmen (Abbildung 1). 


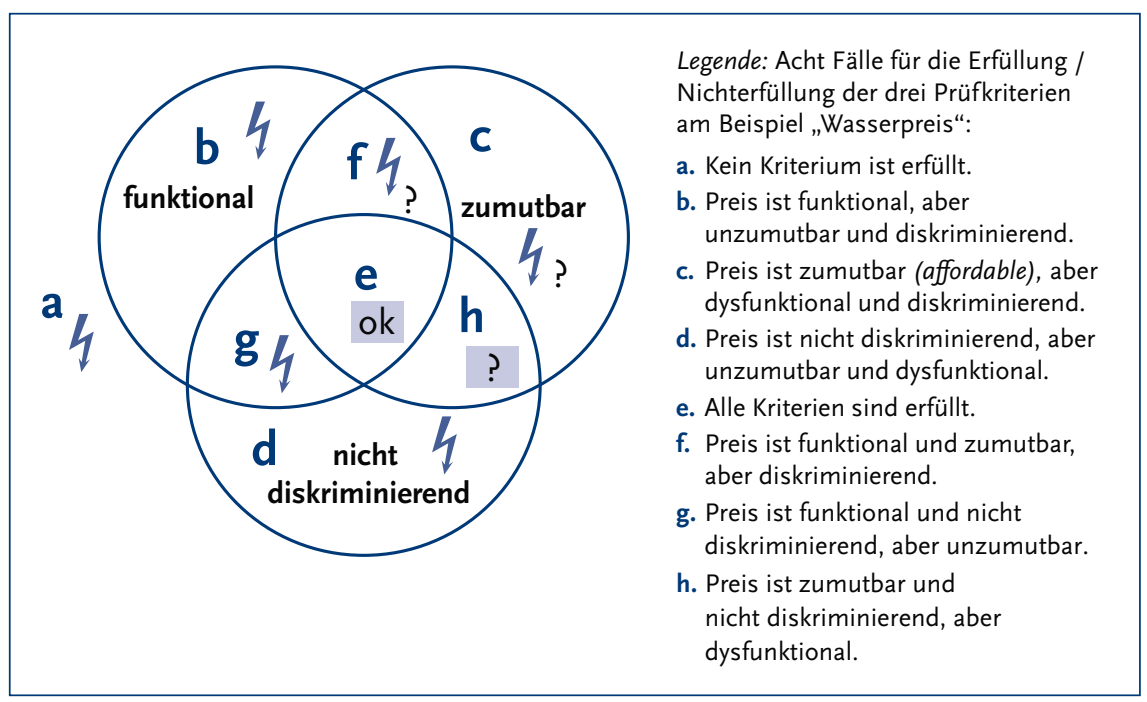

Abbildung 2: Die normativen Prüfkriterien im Mengendiagramm und acht Fallunterscheidungen
Funktionalität verbünden. Unter Funktionalitäts-Gesichtspunkten wird hier die pekuniäre Hürde sogar kritischer geprüft als allein mit dem affordability-Kriterium. Preise können aber auch funktional „zu gering“ sein. Das ist etwa der Fall, wenn Umwelt- und Ressourcenkosten der Wasserentnahme (externe Effekte) ungenügend eingepreist sind. Hier müsste man sich, wenn man sich als Anwalt eines nachhaltigen RaW versteht, grundsätzlich für eine Erhöhung der Hürden einsetzen, um das Feld e zu erreichen. In diesem Fall kann es allerdings auch zu einem trade-off insbesondere zwischen Zumutbarkeit und Funktionalität kommen. Hier würde ein RaW wohl zugunsten der Zumutbarkeit bei der Funktionalität Abstriche machen.
Aus dieser Perspektive ist das RaW dann erfüllt, wenn jedes der drei Kriterien in allen Zugangsdimensionen erfüllt wäre. Bei der Prüfung ist zu beachten, dass sich die Hürdenhöhen prinzipiell über die unterschiedlichen Hürdenarten wie „kommunizierende Röhren“ austarieren lassen - insbesondere auch aufgrund des trade-offs von pekuniären und nicht pekuniären Hürden (zum Beispiel höherer service level gegen höheres Trinkwasser-Entgelt). In der Realität werden die drei Prüfkriterien allerdings vielfach nur teilweise erfüllt sein. Genau genommen sind kombinatorisch sogar acht Fälle möglich (Fälle a bis h in Abbildung 2). Hier stellt sich die Frage, in welchen Fällen das Recht auf Wasser (noch) als erfüllt anzusehen wäre.

Die Fälle a und e bilden die eindeutigen Extreme ab. Im Fall a ist nicht ein einziges Kriterium erfüllt. Im Fall e sind alle Kriterien in der oben geforderten Form erfüllt, damit zweifelsfrei auch ein RaW. Die Fälle b, d und g dürften sich durch mangelnde Zumutbarkeit, die am stärksten etablierte Forderung in der Debatte, disqualifizieren. Die Fälle c und f sind dadurch gekennzeichnet, dass die Hürden zwar zumutbar sind. Allerdings ist die Nichtdiskriminierung nicht gegeben, die als Forderung in der Debatte der letzten Jahre auch eine zunehmend wichtige Rolle spielt. Am interessantesten erscheint der Fall h: Hier sind die Hürden zumutbar und nicht diskriminierend, aber dysfunktional.

\section{Dysfunktionale Hürden}

Dysfunktional können die Hürden in zwei Richtungen sein: „zu hoch“ oder aber „zu gering“. Der Wasserpreis etwa kann funktional „zu hoch“ sein durch die bereits erwähnten Monopolaufschläge. Wohlgemerkt: Trotz solcher Aufschläge kann Wasser für den Haushalt gleichzeitig noch „affordable“ und die Tarifstruktur nicht diskriminierend gestaltet sein. Hier mag man sich als Anwalt des RaW schnell mit dem Kriterium der
Demnach wäre eine Situation wie in Feld h unter Umständen durchaus vertretbar.

\section{Implikationen des Konzepts für die Debatte}

Was bedeutet diese Analyse nun für die aktuelle Debatte um die Implementation eines RaW?

- Es verdient Beachtung, dass durch das zusätzliche Kriterium der Funktionalität die hier formulierten Anforderungen an Hürden - obgleich aus ökonomischer Perspektive generiert - zum Teil sogar strenger ausfallen als jene, die in der bisherigen Debatte prominent diskutiert werden (gestützt auf Zumutbarkeit und Nichtdiskriminierung).

- Aus der Hürden-Betrachtung wird auch eine Rückbindung an die Privatisierungsdebatte möglich. Eine Privatisierung kann dazu führen, dass in der Folge Wasserpreise oberhalb einer funktionalen Höhe liegen („Monopolaufschlag“). Eine solche Verletzung des RaW könnte aber gleichermaßen durch die Ineffizienz und mangelnde Entgeltregulierung eines öffentlichen Versorgers eintreten. Auch die Verringerung des service levels als Folge von Privatisierung ist natürlich vorstellbar (Erhöhung nicht pekuniärer Hürden durch Qualitätseinbußen). Letztlich kommt es aber unabhängig von der Trägerschaft des Versorgers entscheidend darauf an, inwieweit eine staatliche Preis- und Qualitätsregulierung Erfolg hat. Deshalb sind „dem Wesen nach“ Privatisierung der Wasserversorgung und $\mathrm{RaW}$ gerade keine Antagonisten (Gawel/Bretschneider 2012, S. 354 f.), wie es noch immer in Teilen der Debatte unterstellt wird (Kluge/ Scheele 2008, S. 15).

- In der Debatte wird regelmäßig beklagt, dass gerade in Großstädten von Entwicklungsländern das Wasser bei „fliegenden Wasserhändlern“, auf welche die Bewohner informeller Wohngebiete angewiesen sind, z. T. erheblich teurer sei als bei Hausanschlüssen (UNDP 2006, S. 52 f.; Montgo- 
mery 1988; Marvin/Laurie 1999; Simpson 2006, S. 103; Israel 2007). Dieses Phänomen lässt sich jedenfalls teilweise damit erklären, dass trotz eines Hausanschlusses gegebenenfalls beträchtliche zeitliche und qualitative Zugangshürden beim Netz bestehen (Unterbrechungen, Druckschwankungen, Qualitätsprobleme - dazu Vairamoorthy et al. 2008, S. 338; Baisa et al. 2010, S. 1; Kumpel/Nelson 2013). Damit wird der informelle Wassermarkt als (heterogenes) Substitut für alle Stadtbewohner relevant, was mit zu den beklagten Preissteigerungen beitragen dürfte (zu Preiseffekten zum Beispiel Klassert et al. 2015).

\section{Weiterer Forschungs- und Klärungsbedarf}

Für die weitere öffentliche Debatte und die ihr zuarbeitende wissenschaftliche RaW-Forschung ergeben sich vor dem Hintergrund der hier vorgestellten konzeptionellen Überlegungen die folgenden beiden Aufgaben:

In der öffentlichen RaW-Debatte sollte weiterhin die normative Konkretisierung der Kriterien für Zumutbarkeit und Nichtdiskriminierung diskutiert werden. Dabei sollten die positiven Erkenntnisse berücksichtigt werden, die die Wissenschaft hierzu liefern kann (zum Beispiel trade-off-Analyse). Gleichzeitig sollte mit vermeintlich eindeutigen, aber umstrittenen wissenschaftlichen Normierungshilfen (z. B. affordability ratio als Quotient aus Wasserausgaben und Einkommen) zurückhaltend umgegangen werden. Hinsichtlich der Nichtdiskriminierung sollte insbesondere darüber eine Verständigung erfolgen, welche „Diskriminierungen“ als legitim angesehen werden können. Die Ökonomik bietet etwa mit der Funktionalität von Nachhaltigkeitshürden legitime Unterscheidungsgründe an. Eine „absolute Nichtdiskriminierung“ ist in der realen Welt ohnehin nicht zu verwirklichen.

Komplementär sollte die RaW-Forschung die wissenschaftlichen Grundlagen zur politischen Bestimmung der normativen Prüfkriterien Funktionalität, Zumutbarkeit und Nichtdiskriminierung vorantreiben. Zur Funktionalität von Zugangshürden kann Wissenschaft wesentliche Erkenntnisse beisteuern. Zu normativen Fragen der Zumutbarkeit und Nichtdiskriminierung kann Wissenschaft immerhin Folgewirkungen und Zielkonflikte aufdecken und so vor allem vor Fehlurteilen warnen.

\section{Fazit}

Die unlängst verabschiedeten SDGs stellen mit ihrer expliziten Nachhaltigkeitsperspektive einen wichtigen Fortschritt gegenüber den MDGs dar, weil sie sich bewusst dem Spannungsfeld zwischen sozialen Belangen (bezogen auf Wasser, aber etwa auch auf Energie) und Nachhaltigkeitszielen aussetzen. Diese Weiterentwicklung sollte in der RaW-Debatte konsequent nachvollzogen werden. Es geht nun darum, die konzeptionelle Entwicklung eines „aufgeklärten RaW“ fortzuschreiben, die spätestens mit dem GC15 begonnen hat. Weder die SDGs noch die Literatur liefern jedoch bislang eine integrative Kon- zeption, mit der die Umsetzung eines derart auf Nachhaltigkeit programmierten RaWs angeleitet werden kann. Das hier vorgestellte Hürdenkonzept schließt diese Lücke, indem konkurrierende Ziele in die Zugangsdefinition einbezogen werden. Hieraus ergeben sich weitreichende Chancen insbesondere für eine handhabbare Umsetzung eines RaW in die Praxis. Die Erweiterung der Zugangsdefinition um das Kriterium der Funktionalität und die hier vorgestellte Hürdenperspektive führen zu einer präziseren Bewertung von Versorgungssituationen in der Praxis, liefern konkrete Ansatzpunkte zur deren Verbesserung, beziehen potenzielle Widerstände von Anfang an mit ein und lösen so den Anspruch der SDGs ein, soziale und sonstige Nachhaltigkeitsbelange gleichermaßen zu berücksichtigen.

\section{Literatur}

Albuquerque, C. (2011): Report of the Special Rapporteur on the Human Right to Safe Drinking Water and Sanitation. UN Doc A/HRC/18/33. New York, United Nations.

Albuquerque, C./Roaf, V. (2012): On the Right Track: Good Practices in Realising the Right to Water and Sanitation. Im Internet unter: www.ohchr.org/Documents/Issues/Water/BookonGoodPractices_en.pdf

Baillat, A./Schmitz, T./Szabó, M. (2013): Towards a Human Rights Based Water Governance: Challenges for the Post 2015 Thematic Consultations on Water. Geneva, WaterLex.

Baisa, B./Davis, L. W./Salant, S. W./Wilcox, W. (2010): The welfare costs of unreliable water service. In: Journal of Development Economics 92/1. S. 1-12.

Balance, T./Taylor, A. (2005): Competition and Economic Regulation in Water. The Future of the European Water Industry. London, IWA Publishing.

Bartram, J. (2008): Improving on haves and have-nots. In: Nature 452. S. 283-284.

Becker, G. S. (1965): A theory of the allocation of time. In: Economic Journal 75/299. S. 493-516.

CESCR (Committee on Economic, Social and Cultural Rights) (2002): General Comment No. 15. The Right to Water (Arts. 11 and 12 of the International Covenant on Economic, Social and Cultural Rights). Geneva.

Dubreuil, C. (2006): The Right to Water: From Concept to Implementation. Marseilles, World Water Council.

Flores, Ó./Jiménez, A./Pérez-Foguet, A. (2013): Monitoring access to water in rural areas based on the human right to water framework: A local level case study in Nicaragua. In: International Journal of Water Resources Development 29/4. S.605-621.

Gawel, E./Bretschneider, W. (2012): Recht auf Wasser - eine institutionenökonomische Perspektive. In: ORDO. Jahrbuch für die Ordnung von Wirtschaft und Gesellschaft 63. S. 325-363.

Gawel, E./Sigel, K./Bretschneider, W. (2013): Affordability of water supply in Mongolia: Empirical lessons for measuring affordability. In: Water Policy 15/1. S. 19-42.

Gorsboth, M./Wolf, E. (2008): Identifying and Adressing Violations of the Human Right to Water. Applying the Human Rights Approach. Stuttgart, Bread for the World.

Hancock, K. E. (1993): ‘Can pay? Won't pay?’ or economic principles of 'affordability'. In: Urban Studies 30/1. S. 127-145.

Howard, G./Bartram, J. (2003): Domestic Water Quantity: Service Level and Health. Geneva, World Health Organization.

Israel, D. K. (2007): Impact of increased access and price on household water use in urban Bolivia. In: The Journal of Environment and Development 16/1. S. 58-83.

Klassert, Chr./Sigel, K./Gawel, E./Klauer, B. (2015): Modeling residential water consumption in Amman: The role of intermittency, storage, and pricing for piped and tanker water. In: Water 7/7. S. 3643-3670. 
Kluge, Th./Scheele, U. (2008): Zwischen Wirtschaftsgut und Menschenrecht: Wasserversorgung und Millennium-Ziele. In: Franzke, J. (Hrsg.): Wasser. Zukunftsressource zwischen Menschenrecht und Wirtschaftsgut, Konflikt und Kooperation. Potsdam. S. 13-27.

Kumpel, E./Nelson, K. L. (2013): Comparing microbial water quality in an intermittent and continuous piped water supply. In: Water Research 47/14. S. 5176-5188.

Lu, Y./Nakicenovic, N./Visbeck, M./Stevance, A.-S. (2015): Five priorities for the UN Sustainable Development Goals. In: Nature 520/7548. S. 432-433.

Marvin, S./Laurie, N. (1999): An emerging logic of urban water management, Cochamba, Bolivia. In: Urban Studies 36. S. 341-357.

Meier, B. M./Kayser, G. L./Amjad, U. Q./Bartram, J. (2013): Implementing an evolving human right through water and sanitation policy. In: Water Policy 15/1. S. 116-133.

Meier, B. M./Kayser, G. L./Kestenbaum, J. G./Amjad, U. Q./Dalcanale, F./Bartram, J. (2014): Translating the human right to water and sanitation into public policy reform. In: Science and Engineering Ethics 20/4. S. 833-848.

Montgomery, J. D. (1988): The informal service sector as an administrative resource. In: Rondinelli, D. A./Cheema, G. Sh. (Hrsg.): Urban Services in Developing Countries. Macmillan. S. 89-111.

Murthy, Sh. L. (2013): The human right(s) to water and sanitation: History, meaning, and the controversy over-privatization. Berkeley Journal of International Law 31/1. S. 89-147.

OECD (Organisation for Economic Co-operation and Development) (2010): Pricing Water Resources and Water and Sanitation Services. Paris.

Roaf, V./Khalfan, A./Langford M. (2005): Monitoring Implementation of the Right to Water: A Framework for Developing Indicators. In: Heinrich Böll Foundation (Hrsg.): Global Issue Paper No. 14.

Sacher, D./Windfuhr, M. (2008): The debate on "water as a human right" and its implications for development assistance. In: Scheumann, W./ Neubert, S./Kipping M. (Hrsg.): Water Politics and Development Cooperation. Local Power Plays and Global Governance. Springer. S. 147-176.

Simpson, R. (2006): Universal access in the water and sanitation sector. In: OECD/World Bank (Hrsg.): Liberalisation and Universal Access to Basic Services: Telecommunications, Water and Sanitation, Financial Services, and Electricity. OECD, S. 97-134.

Singh, N. (2013): Translating human right to water and sanitation into reality: A practical framework for analysis. Water Policy 15/6. S. 943-960.

Thielbürger, P. (2015): Re-conceptualizing the human right to water: A pledge for a hybrid approach. Human Rights Law Review 15/2. S. 225-249.

UN (United Nations) (2015): Transforming Our World: The 2030 Agenda for Sustainable Development. Im Internet unter: sustainabledevelopment.
un.org/content/documents/7891TRANSFORMING\%2OOUR\%2O WORLD.pdf

UN (United Nations) (2015): The Millennium Development Goals Report 2015. New York.

UNDP (United Nations Development Programme) (2006): Human development report 2006. Beyond scarcity: Power, poverty and the global water crisis, New York.

UNESCO (United Nations Educational, Scientific and Cultural Organization) (2015): The United Nations World Water Development Report 2015: Water for a Sustainable World. Paris.

UNICEF (United Nations Children's Fund)/WHO (World Health Organization) (2015): Progress on Sanitation and Drinking Water. 2015 Update and MDG Assessment. Geneva.

Vairavamoorthy, K./Gorantiwar, S. D./Pathirana, A. (2008): Managing urban water supplies in developing countries - Climate change and water scarcity scenarios. In: Physics and Chemistry of the Earth 33/5. S. 330-339.

WHO (World Health Organization)/UNICEF (United Nations International Children's Emergency Fund) (2012): Background-Note on MDGs, NonDiscrimination and Indicators in Water and Sanitation. Im Internet unter: www.wssinfo.org/fileadmin/user_upload/resources/END-BackgroundPaper.pdf

\section{AUTOREN + KONTAKT}

Dr. Erik Gawel ist Professor für Volkswirtschaftslehre an der Universität Leipzig und Leiter des Departments Ökonomie am Helmholtz-Zentrum für

Umweltforschung - UFZ.

Helmholtz-Zentrum für Umweltforschung - UFZ, Department Ökonomie, Permoser Str. 15, 04318 Leipzig.

Tel.: +49 341 235-1940, E-Mail: erik.gawel@ufz.de

Wolfgang Bretschneider ist wissenschaftlicher Mitarbeiter am Department Ökonomie des UFZ.
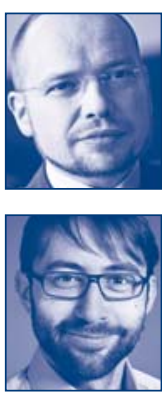

Helmholtz-Zentrum für Umweltforschung - UFZ, Department Ökonomie, Permoser Str. 15, 04318 Leipzig.

Tel.: +49 341 235-1690,

E-Mail: wolfgang.bretschneider@ufz.de

\section{Nachhaltigkeit}

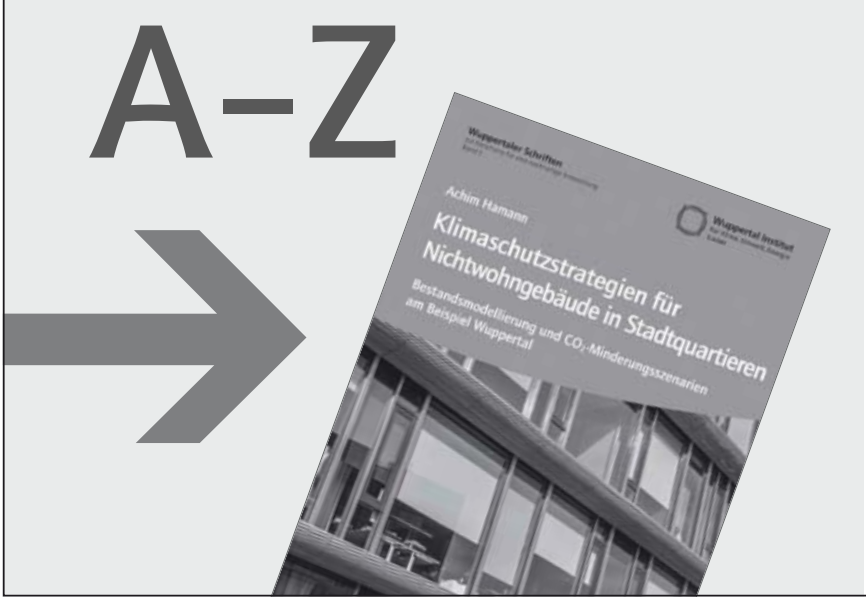

\section{E wie Entdeckung}

Sie tragen die Nichtbeachtung schon im Namen: Sogenannte Nichtwohngebäude sind über ihre Raumwärme für rund 13 Prozent der $\mathrm{CO}_{2}$-Gesamtemissionen verantwortlich. Doch im Gegensatz zu Wohngebäuden werden Bürogebäude, Fabrikhallen, Handelsflächen und Kliniken bisher in Untersuchungen und Prognosen stark vernachlässigt. Dieses Buch bemisst nicht nur deren aktuelle Emissionen, sondern schildert auch Sanierungspotenziale und $\mathrm{CO}_{2}$-Minderungsszenarien bis zum Jahr 2050 .

\section{Achim Hamann}

Klimaschutzstrategien für Nichtwohngebäude in Stadtquartieren Bestandsmodellierung und $\mathrm{CO}_{2}$-Minderungsszenarien am Beispiel Wuppertal 312 Seiten, broschiert, 34,95 Euro, ISBN 978-3-86581-699-3

\section{Erhältlich bei}

www.oekom.de | oekom@verlegerdienst.de

Die guten Seiten der Zukunft 